\title{
The structural properties of imidazole cured epoxy-phenol resins
}

\author{
Yi-Cheng Chen, Wen-Yen Chiu* \\ Department of Chemical Engineering, National Taiwan University, Taipei 10617, Taiwan, ROC
}

Received 28 March 2000; received in revised form 20 June 2000; accepted 27 December 2000

\begin{abstract}
In this work, structural properties of the imidazole cured epoxy-phenol resins were theoretically simulated by the Monte Carlo (MC) procedure. The proposed procedure can be extended to any other complicated reaction system. The structural properties, including the epoxide conversion, the weight average molecular weight, the sol-gel fraction, the number average branch point of the sol part, the branch as well as the loop density of the gel part were discussed. The theoretical results were further verified experimentally by differential scanning calorimetry (DSC), sol extraction test, and gel permeation chromatography (GPC). The theoretical results agree with the experimental data quite well. (C) 2001 Elsevier Science Ltd. All rights reserved.
\end{abstract}

Keywords: Monte Carlo simulation; Epoxy resins; Structural properties

\section{Introduction}

The unique chemical and physical properties of epoxyphenol resins have made them the predominated thermosetting resins for potting and encapsulating a variety of electrical and electronic components [1-3]. Nowadays the chip sizes have become larger, while the dimensions of devices inside the chip have become smaller and the structure of the chip has become more delicate. Encapsulants formulated out of epoxy-phenol resins are transfer-molded to form a protective shell outside the integrated circuits. During polymerization, the molecular weight increases and the crosslinked structure builds up, which governs the rheological and mechanical properties of the cured resins. The polymerization of diglycidyl ether of bisphenol A (DGEBA) cured with bisphenol A (BPA) and 2-ethyl-4-methyl-imidazole $(2,4 \mathrm{EMI})$, forming a liner structure or a densely crosslinked network, is controlled by the molar ratio between DGEBA and BPA. There have been some studies on the reaction kinetics [4-7]; however, a theoretical treatment on the chain build-up and network formation governed by these apparent complicated reaction mechanisms is still lacking.

In our previous studies [8], a generalized Monte Carlo (MC) procedure was proposed, which could provide a very detailed insight information about the structural evolution of polymer chains. This it is very important for an

\footnotetext{
* Corresponding author. Tel.: +886-2-2362-3259; fax: +886-2-23623040.

E-mail address: ycchiu@ms.cc.ntu.edu.tw (W.-Y. Chiu).
}

understanding of the chemorheological properties due to the growing up of branched (or crosslinked) structures inside the curing polymers.

In this work, the epoxide conversion, the weight average molecular weight and the number average branch point of the sol part, the sol-gel fraction, as well as the branch and loop density of the gel part were studied by the proposed MC method. The theoretical results were verified experimentally by differential scanning calorimetry (DSC) and gel permeation chromatography (GPC). Both theoretical and experimental results coincided with each other very well.

\section{Experimental}

\subsection{Materials}

The epoxy resin (DER332), a nearly pure form of DGEBA, was supplied by the Dow Chemicals Company. The epoxide equivalent weight (EEW) of DER332, determined by titration, was $174 \mathrm{~g} / \mathrm{mol}$-epoxide. The difunctional phenol, BPA with a purity $>99 \%$, was obtained from the Tokyo Chemical Industry Company. The imidazole, 2,4EMI with a purity $>99 \%$, was obtained from Acros Organics. All materials were stored in a desiccator and used as received without further purification.

\subsection{Sample preparation}

Different molar ratios of BPA-epoxide and 2,4EMI were 


\section{Initiated by $1: 1$ adduct \& $2: 1$ adduct}

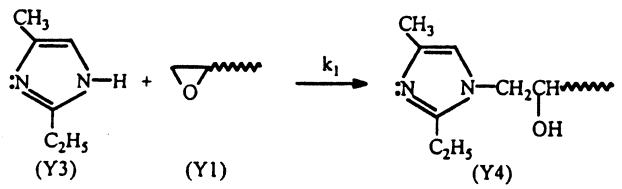

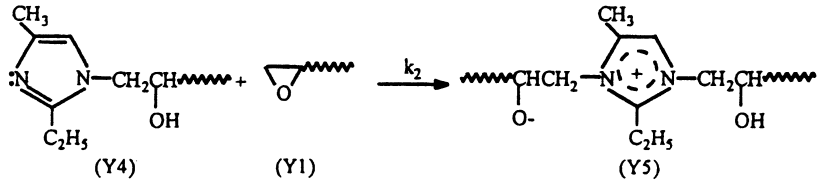

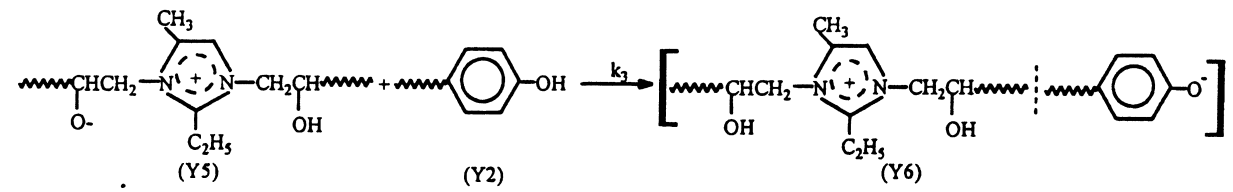

IU Initiated by 2,4 EMI \& BPA Complex<smiles>Cc1c[nH]c(O)n1</smiles>

(Y3) (Y2)

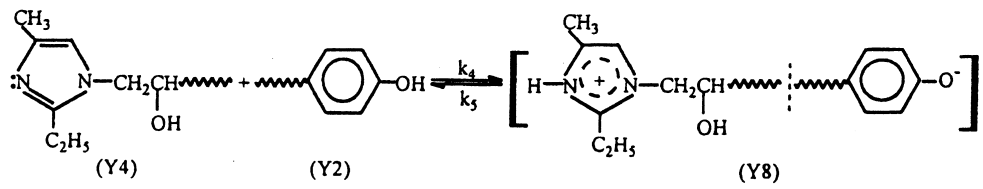

\section{Propagation}

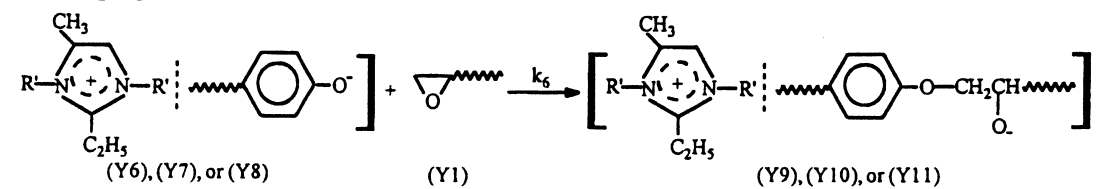

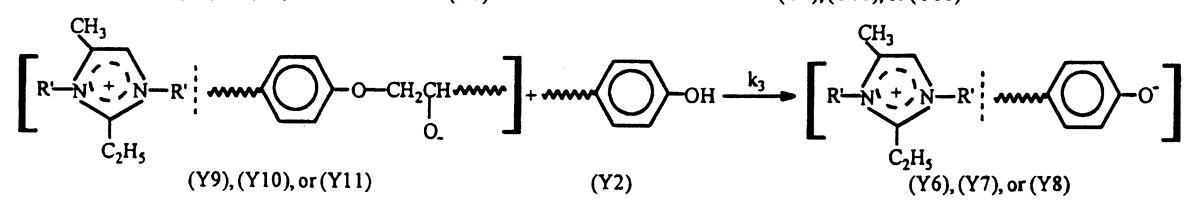
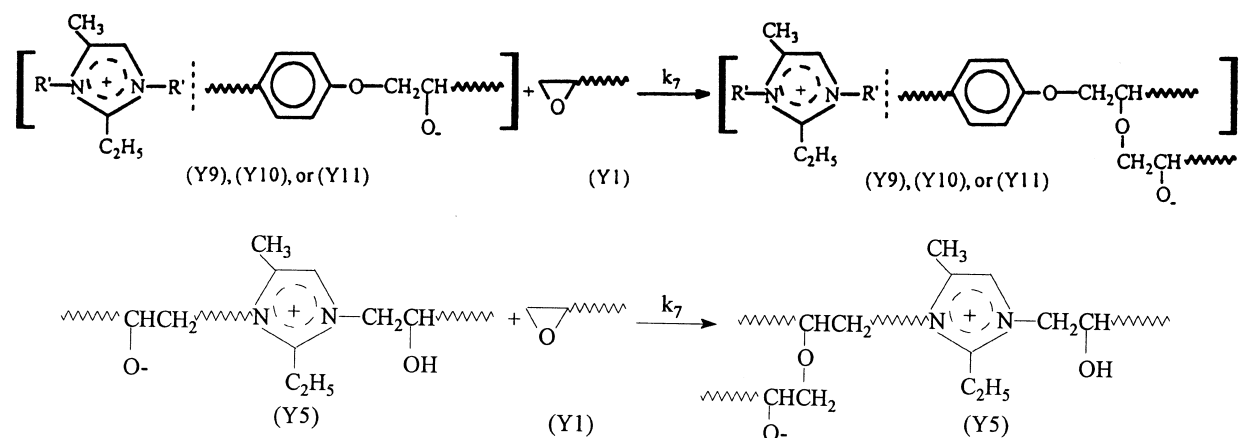

where $\mathrm{R}^{\prime}: \mathrm{H}$ or $\mathrm{CH}_{2} \mathrm{CH}$ mm

$\mathrm{OH}$

Fig. 1. Reaction mechanisms proposed for DGEBA cured with BPA and 2,4EMI. 
dissolved separately in spectrometric grade acetone. The solutions were then mixed well by rigorous stirring, and the acetone was evaporated under vacuum at $30^{\circ} \mathrm{C}$ for $10 \mathrm{~min}$. The resultant mixture was immersed in ice water immediately to minimize further cure reaction before kinetics studies and other experiments. The prepared samples were surveyed from room temperature to $320^{\circ} \mathrm{C}$ by thermogravimetric analysis (TGA). The results of TGA showed that the total weight loss caused by residual acetone loss and thermal degradation of resultant mixture is less than $1 \%$.

\subsection{Polymerization and sol extraction}

The prepared samples (ca. $5 \mathrm{~g}$ ) were poured into empty glass tubes and sealed well. All sample tubes were immersed into an isothermal silicon oil bath, and then the further reaction restarted at the setting temperature. The sample tubes were removed from the silicon oil bath at various reaction-time intervals, quenched immediately and pulverized in liquid nitrogen. Tetrahydrofuran (THF) was then added into the sample tube to extract the soluble portion from the cured sample. All sample tubes were fixed on a shaker water bath and kept at $15^{\circ} \mathrm{C}$ during the extraction experiments. The sample was extracted for at least two days and the solvent was replaced with fresh THF every six hours. The sol fraction was determined from the difference between the initial and final weights of the cured sample.

\subsection{Gel permeation chromatography (GPC) analysis}

A Waters Associates Chromatograph (Model 6000A pump) was equipped with three columns, one Polymer Laboratories Mixed-C and two American Polymer Standard $100 \AA$ in series, connected to the Shodex RI-71 refractive index detector. The analytically pure THF was used as eluent and the flow rate was set to $1 \mathrm{ml} / \mathrm{min}$. The average molecular weights were determined from the calibration curve established by means of polystyrene (PS) standards (Polymer Laboratories) with normal molecular weights 162 , 580, 2100, 3250, 9200, 28,500, 66,000, 156,000, 330,000, $1,030,000$, and 3,040,000.

\subsection{Differential scanning calorimetry (DSC) analysis}

Both dynamic and isothermal DSC were performed by using a TA Instrument (TA-2010) DSC under nitrogen atmosphere. The DSC enthalpy and temperature scale was calibrated using a high purity indium sample. Dynamic scans from 2.5 to $20^{\circ} \mathrm{C} / \mathrm{min}$ over a temperature range of $25-320^{\circ} \mathrm{C}$ were used to cure the 2,4EMI-BPA-epoxide samples. Isothermal studies were also performed at the desired temperature, held for various periods of time. Both dynamic and isothermal samples were quenched to $-40^{\circ} \mathrm{C}$ and scanned up to $300^{\circ} \mathrm{C}$ at a heating rate of $20^{\circ} \mathrm{C} /$ min to detect the residual heat of reaction. The post-cured sample was then quenched to $0^{\circ} \mathrm{C}$ and scanned at $20^{\circ} \mathrm{C} / \mathrm{min}$ to detect the glass transition temperature $\left(T_{\mathrm{g}}\right)$.

\section{Theoretical approach}

\subsection{Reaction mechanisms}

The proposed reaction mechanisms are shown in Fig. 1 on the basis of our previous studies [7]. It shows two initiation mechanisms: the first one starts with the adduct ion of the pyridine-type nitrogen with epoxy $(1.1,1.2)$. The second results from the ionic nature of the 2,4EMI-phenol interaction $(1.4,1.5)$. And the following propagation steps consist of three main reaction mechanisms: the epoxide-phenol reaction (1.6), the acid-base reaction (1.3, 1.7), and the epoxide- $\mathrm{R}-\mathrm{O}^{-}$reaction $(1.8,1.9)$. And the corresponding mathematical scheme can be expressed as: (where ' $y_{i}$ ' is identified in Fig. 1)

$$
\begin{aligned}
\frac{\mathrm{d} y_{1}}{\mathrm{~d} t}= & -k_{1} y_{1} y_{3}-k_{2} y_{1} y_{4}-k_{6} y_{1}\left(y_{6}+y_{7}+y_{8}\right) \\
& -k_{7} y_{1}\left(y_{5}+y_{9}+y_{10}+y_{11}\right) \\
\frac{\mathrm{d} y_{2}}{\mathrm{~d} t}= & -k_{3} y_{2} y_{5}-k_{4} y_{2}\left(y_{3}+y_{4}\right)+k_{5}\left(y_{7}+y_{8}\right) \\
& -k_{3} y_{2}\left(y_{9}+y_{10}+y_{11}\right)
\end{aligned}
$$

$\frac{\mathrm{d} y_{3}}{\mathrm{~d} t}=-k_{1} y_{1} y_{3}-k_{4} y_{2} y_{3}+k_{5} y_{7}$

$\frac{\mathrm{d} y_{4}}{\mathrm{~d} t}=k_{1} y_{1} y_{3}-k_{2} y_{1} y_{4}-k_{4} y_{2} y_{4}+k_{5} y_{8}$

$\frac{d y_{5}}{d t}=k_{2} y_{1} y_{4}-k_{3} y_{2} y_{5}$

$\frac{\mathrm{d} y_{6}}{\mathrm{~d} t}=k_{3} y_{2}\left(y_{5}+y_{9}\right)-k_{6} y_{1} y_{6}$

$\frac{\mathrm{d} y_{7}}{\mathrm{~d} t}=k_{4} y_{2} y_{3}-k_{5} y_{7}-k_{6} y_{1} y_{7}+k_{3} y_{2} y_{10}$

$\frac{\mathrm{d} y_{8}}{\mathrm{~d} t}=k_{4} y_{2} y_{4}-k_{5} y_{8}-k_{6} y_{1} y_{8}+k_{3} y_{2} y_{11}$

$\frac{\mathrm{d} y_{9}}{\mathrm{~d} t}=k_{6} y_{1} y_{6}-k_{3} y_{2} y_{9}$

$\frac{\mathrm{d} y_{10}}{\mathrm{~d} t}=k_{6} y_{1} y_{7}-k_{3} y_{2} y_{10}$

$\frac{\mathrm{d} y_{11}}{\mathrm{~d} t}=k_{6} y_{1} y_{8}-k_{3} y_{2} y_{11}$ 
Table 1

Reaction rate constants [7] used in the MC simulation $\left(k_{1}\right.$ and $k_{2}(1 / \mathrm{mol} \mathrm{s}): \ln (A) 18.33, E_{\mathrm{a}}(\mathrm{kJ} / \mathrm{mol}) 74 ; k_{3}(1 / \mathrm{mol} \mathrm{s}): \ln (A) 13.52, E_{\mathrm{a}}(\mathrm{kJ} / \mathrm{mol}) 38.35 ; K_{4}(1 / \mathrm{mol} \mathrm{s})$ $5 \times 10^{-2} ; k_{5}\left(1 / \mathrm{s} 10^{-7}\right.$.) For $k_{6}$ and $k_{7}$ values are tabulated below

\begin{tabular}{|c|c|c|c|c|c|c|}
\hline \multirow{2}{*}{$\begin{array}{l}\text { Molar ratio } \\
\text { DGEBA/ } \\
\text { BPA/2,4EMI }\end{array}$} & \multicolumn{3}{|c|}{$k_{6}(1 / \mathrm{mol} \mathrm{s})$} & \multicolumn{3}{|c|}{$k_{7}(1 / \mathrm{mol} \mathrm{s})$} \\
\hline & $\ln (A)$ & $E_{\mathrm{a}}(\mathrm{kJ} / \mathrm{mol})$ & $R$ & $\ln (A)$ & $E_{\mathrm{a}}(\mathrm{kJ} / \mathrm{mol})$ & $R$ \\
\hline $100 / 40 / 6$ & 20.40 & 79.81 & -0.998 & 21.46 & 80.07 & -0.997 \\
\hline $100 / 40 / 4$ & 20.62 & 80.18 & -1.000 & 20.73 & 77.36 & -0.992 \\
\hline $100 / 80 / 4$ & 21.35 & 81.42 & -0.998 & 20.35 & 81.42 & -0.998 \\
\hline $100 / 100 / 6$ & 20.68 & 79.34 & -0.998 & 21.68 & 79.34 & -0.998 \\
\hline $100 / 100 / 4$ & 20.69 & 79.30 & -0.997 & 20.69 & 79.30 & -0.997 \\
\hline
\end{tabular}

\subsection{Monte Carlo procedure}

If a system consists of ' $p$ ' irreversible reactions (the reversible reaction can be separated into two elementary

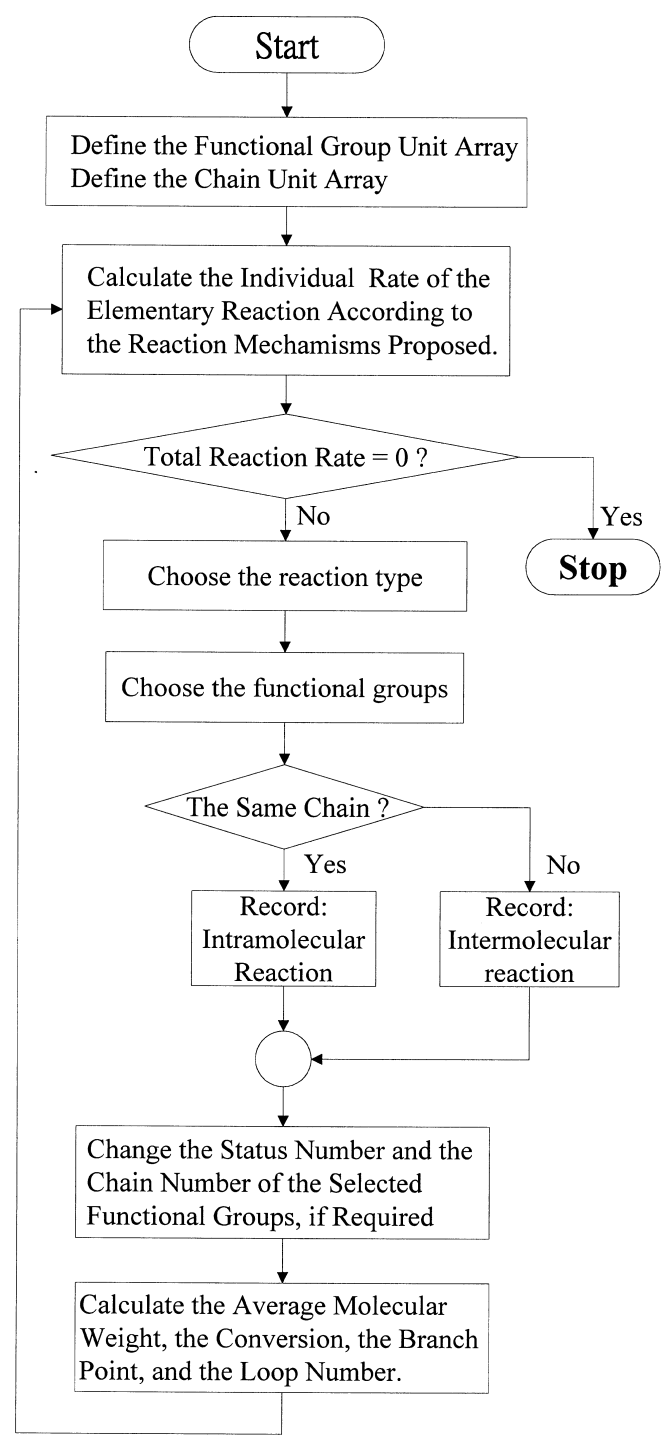

Fig. 2. A concise flow-chart of the Monte Carlo simulation. ones), the general form can be written as follows:

$Y_{m 1}+Y_{m 2} \stackrel{k_{m}}{\rightarrow} Y_{m 3}+Y_{m 4}$

For $m$ th reaction $R_{m}(m=1, \ldots, p)$

where $Y_{m i}$ represents the species $Y_{i}$ participating in the $m$ th reaction, $k_{m}$ denotes the reaction rate constant of $m$ th reaction. The reaction rate equation of the elementary reaction could be expressed as

$r_{m}=k_{m} C_{1} C_{2}$,

where $r_{m}$ is the reaction rate of $m$ th reaction $R_{m}$ and $C_{i}$ is the molar concentration of species $i$.

The strategy of the MC method is divided into two steps: the first is to decide the type of the reaction mechanism, and the second is to select the reacting functional groups [9]. A concise flowchart is shown in Fig. 2. All parameters used in the MC simulation are listed in Table 1 . Since the average value of $n$ of DER332 is 0.028 , the epoxy prepolymer is assumed to be a mixture having $97.2 \mathrm{~mol} \%$ of pure DGEBA $(n=0)$ and $2.8 \mathrm{~mol} \%$ of DGEBA $(n=1)$. Their molecular weights are listed in Table 2. The results of the MC simulation shown in the following figures are the mean values, with at least five times independent of MC calculation.

\section{Results and discussion}

According to the method of reduced average molecular size [10], the critical conversion (or the gel point) is defined as the conversion at which the weight average molecular weight, excluding the largest molecule, reaches the maximum value. The molecular weight profiles of the 1 st, 2 nd,

Table 2

The molecular weight of reactants used in MC simulation

\begin{tabular}{ll}
\hline Material & Molecular weight $(\mathrm{g})$ \\
\hline DGEBA $(n=0)$ & 340 \\
DGEBA $(n=1)$ & 624 \\
BPA & 228 \\
2,4 EMI & 110 \\
\hline
\end{tabular}




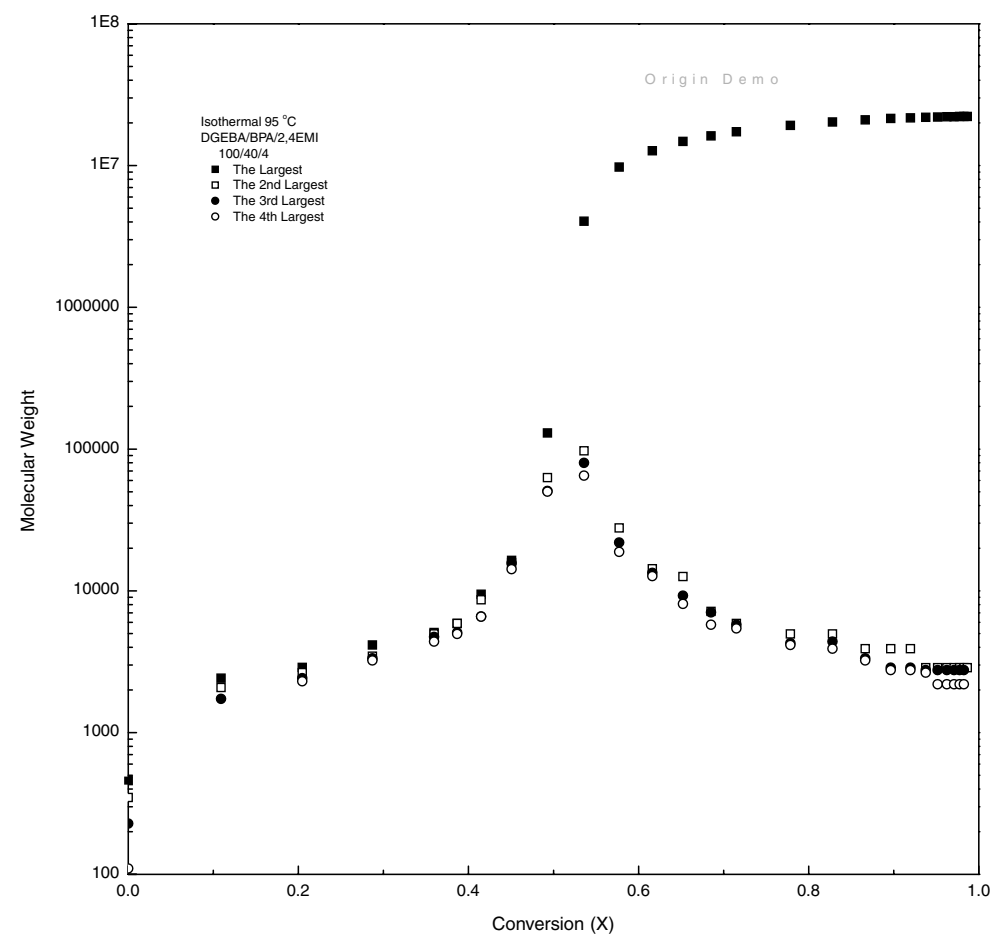

Fig. 3. The weight profile vs. conversion of the 1st, 2nd, 3rd, 4th, largest molecule simulated by the MC method, at a molar ratio DGEBA/BPA/2,4EMI: 100/ $40 / 4$, at $100^{\circ} \mathrm{C}$.

3rd, and 4th largest molecules calculated by the MC method $(N=100,000)$ are plotted in Fig. 3. Before the gel point, all the four molecules almost have the same molecular weight. Their molecular weights rise up steeply as the conversion increases near the gel point. After the conversion exceeds the critical conversion, only the first largest molecule keeps growing, while the others decrease in their molecular weight. This phenomenon is straightforward according to kinetic theory, because large molecules with large number of functional groups are much easier to combine together than the smaller ones. The size effects of MC simulation on the critical epoxy conversion are listed in Table 3 . When the size is large enough (e.g. $N=100,000)$, the critical conversion of the finite system converges and the standard deviation becomes small.

\subsection{Conversion}

Because the amount of heat evolved during the reaction is

Table 3

Size effect on the critical epoxide conversion. The molar ratio of DGEBA/ BPA/2,4EMI was $100 / 40 / 4$ and the reaction temperature was $100^{\circ} \mathrm{C}$. The standard deviation was calculated with at least five times of independent MC simulation

\begin{tabular}{lll}
\hline No. of epoxide groups & Critical conversion & Standard deviation \\
\hline 100 & 0.541 & 0.035 \\
1000 & 0.523 & 0.028 \\
10,000 & 0.514 & 0.012 \\
100,000 & 0.512 & 0.002 \\
\hline
\end{tabular}

related to the number of reacted epoxide molecules [11-12], the conversion of epoxide groups can be expressed as:

$X(t)=\Delta H(t) / \Delta H_{\text {total }}$,

where $X(t)$ is the thermal conversion at time $t, \Delta H_{\text {total }}$ is the total heat of reaction determined from the dynamic DSC studies [7], and $\Delta H(t)$ is the heat of reaction from initial time to the time $t$ during the isothermal reaction. The total heat of reaction used here is listed in Table 4.

A comparison of the epoxide conversion vs. time by the MC simulation and experimental results are shown in Fig. 4. Within $70 \%$ conversion, i.e. $\ln (1-X)>-1.2$, it is found that the prediction by the MC method coincides with the experimental results very well. For DGEBA cured with $100 \%$ molar ratio of BPA, there are enough phenols to consume with epoxide groups through the epoxide-phenol reaction and form linear aromatic ether linkages with

Table 4

The total heat of reaction $\left(\Delta H_{\text {total }}\right)$ and the glass transition temperature $\left(T_{\mathrm{g}}\right)$ for different molar ratio of DGEBA/BPA/2,4EMI [7]

\begin{tabular}{llc}
\hline $\begin{array}{l}\text { Molar ratio } \\
\text { (DGEBA/BPA/ }\end{array}$ & $\Delta H_{\text {total }}(\mathrm{kJ} /$ mol-epoxide $)$ & $T_{\mathrm{g}}\left({ }^{\circ} \mathrm{C}\right)$ \\
2,4EMI) & & \\
\hline $100 / 100 / 6$ & 77.288 & 69.7 \\
$100 / 100 / 4$ & 78.709 & 72.2 \\
$100 / 80 / 4$ & 79.169 & 79.8 \\
$100 / 40 / 6$ & 79.127 & 96.7 \\
$100 / 40 / 4$ & 81.761 & 106.0 \\
\hline
\end{tabular}




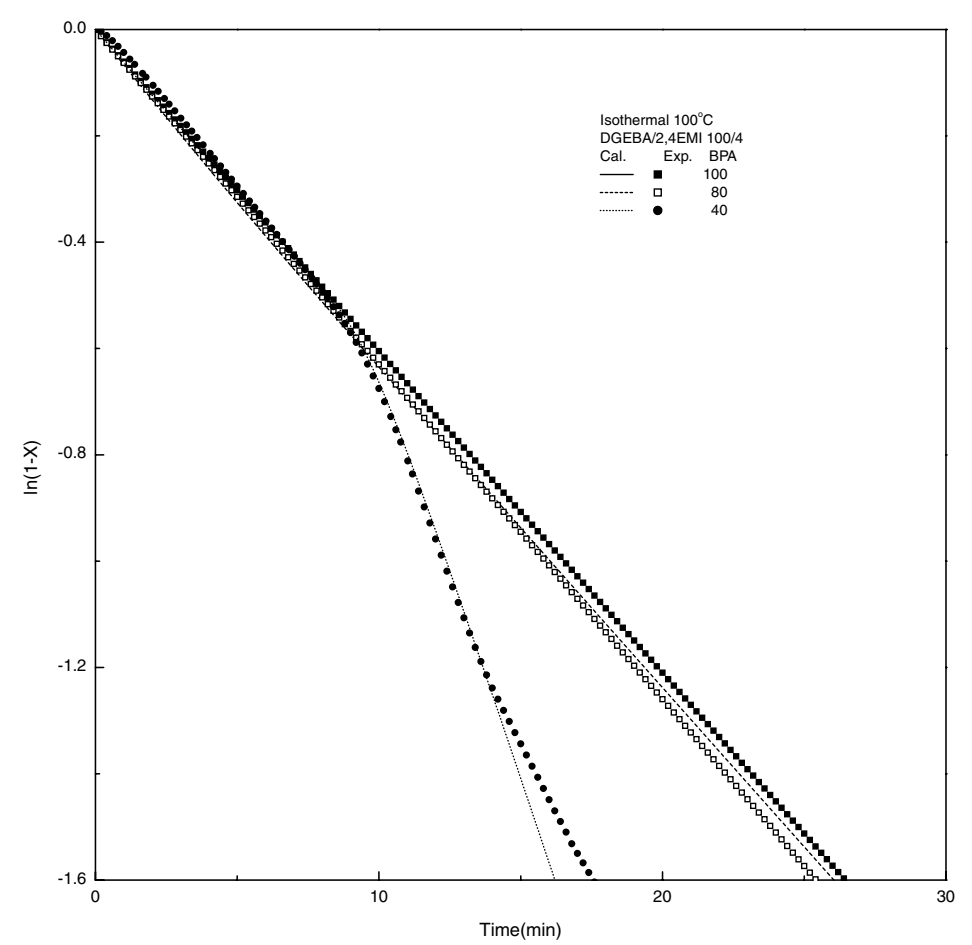

Fig. 4. The comparison of conversion vs. time calculated by the MC method and experimental data by DSC for DGEBA cured with 4\% molar ratio of 2,4EMI and different BPA molar ratios at $100^{\circ} \mathrm{C}$.

secondary hydroxyl groups. As the BPA concentration is lowered, some epoxide groups are still unreacted after the initial epoxide-phenol reaction. The remaining epoxides then react with $\mathrm{RO}^{-}$and form aliphatic ether linkages, from which the branched structure builds up. Because the reaction rate of epoxide- $\mathrm{R}-\mathrm{O}^{-}$is faster than that of epoxide-phenol [7], there is an obvious transition form the initial slope to a steeper one in the conversion-time curve as the BPA molar ratio is $40 \%$. If the BPA molar ratio is $100 \%$, there is no obvious diffusion effect before the conversion reaches $80 \%$, i.e. $\ln (1-X)>-1.6$. On the other hand, there comes a deviation between the MC results and the experimental data at a high conversion interval if the BPA molar ratio is $40 \%$ due to the serious diffusion effect, as shown in Fig. 4.

\subsection{Sol-gel fraction}

In the MC simulation, the calculated gel fraction is defined as the weight of the first largest molecule over the total weight of the curing system. A comparison of the theoretical and the experimental gel fraction vs. conversion is shown in Fig. 5. When the BPA molar ratio is $100 \%$, no gel part is found at any reaction temperature or at molar ratio of 2,4EMI; both theoretical and experimental results agree with each other. When the BPA molar ratio is less than $100 \%$, the gel part forms after the phenol groups are nearly consumed and the weight fraction of the gel part rises up sharply. The profiles of gel fraction are a strong function of the BPA molar ratio. If a less molar ratio of BPA is used initially, the BPA molecules are depleted earlier and the gel point occurs at a lower epoxide conversion. It is also found that either the reaction temperature or the molar ratio of 2,4EMI has only a slight influence on the profiles of gel fraction.

\subsection{Branch point of sol}

On the basis Flory's works [13], the aliphatic ether linkage $(\mathrm{R}-\mathrm{O}-\mathrm{R})$, which is formed through the epoxide- $\mathrm{R}-\mathrm{O}^{-}$reaction, is termed as the 'branch point' in the studies. And the 'loop' is defined as the structure formed by the intra-chain reaction. According to our simulation results, very few loop structures were found in the sol part during the MC calculation, no matter what initial conditions were chosen.

The number average branch point per polymer chain vs. conversion is shown in Fig. 6, when the BPA molar ratio is $100 \%$. It is found that the average value of branch point is very small. The results agree with our hypothesis: if the BPA molar ratio is $100 \%$, there are enough phenol groups to react with epoxide groups, which results in a linear aromatic ether linkage, $\mathrm{Ar}-\mathrm{O}-\mathrm{C}$. It shows that neither the temperature nor the molar ratio of 2,4EMI affect the tendencies significantly. And the profiles of branch point rise up with increasing the conversion. When the BPA molar ratio is lowered to $40 \%$, the profiles of the branch point of sol part reach a maximum value at a critical conversion $(X=$ 0.512), as shown in Fig. 7. It seems that the branch point is also an index of the gel formation. The gel part is formed as soon as the average branch point profile rises up sharply with the increasing conversion. 


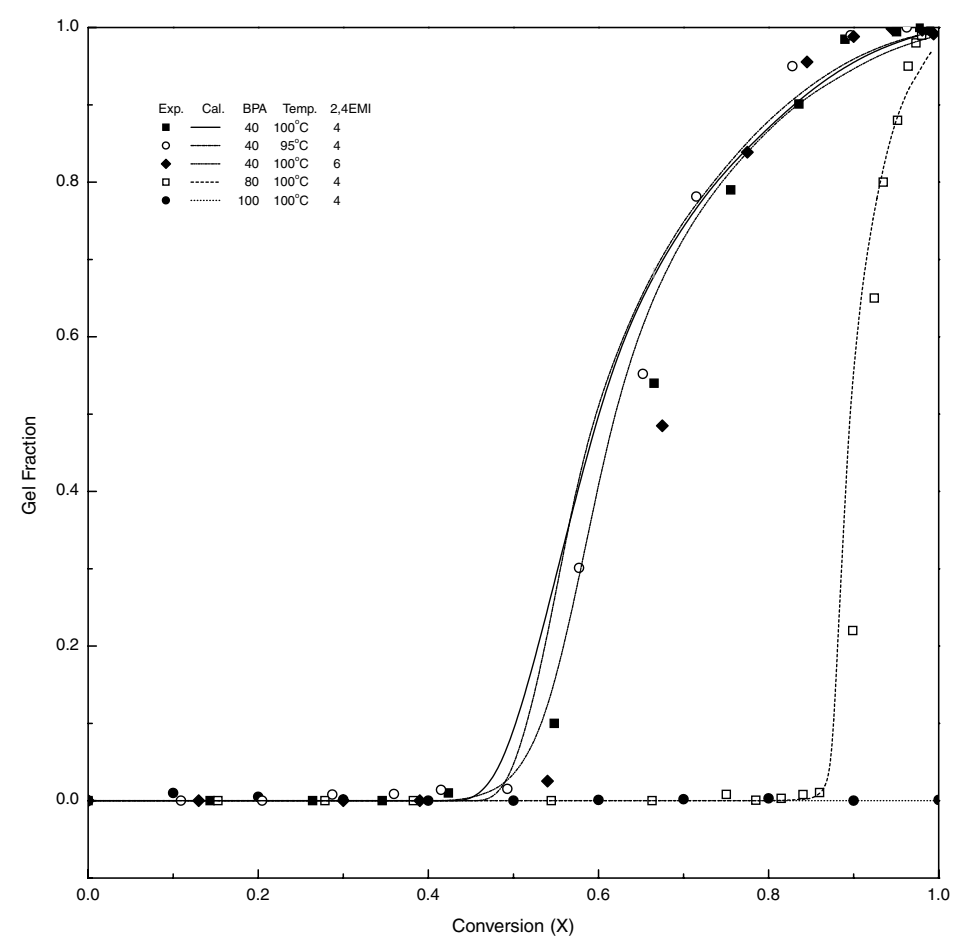

Fig. 5. The gel fraction profile vs. conversion for DGEBA cured with different molar ratios of 2,4EMI and BPA, as well as different temperatures.

\subsection{Molecular weight and glass transition temperature}

Because the GPC columns are calibrated by PS standards, some transformations are needed to compare the theoretical and experimental results. In our previous work [8,9], the weight average molecular weight calculated theoretically by the MC was defined as the absolute molecular weight $\left(\bar{M}_{\mathrm{w}}\right)$ and the value determined from GPC was termed as $\left(\bar{M}_{\mathrm{GPC}}\right)$. If the branched polymers were considered, the calculated $\bar{M}_{\mathrm{w}}$ was, chain by chain, transformed into the reduced molecular

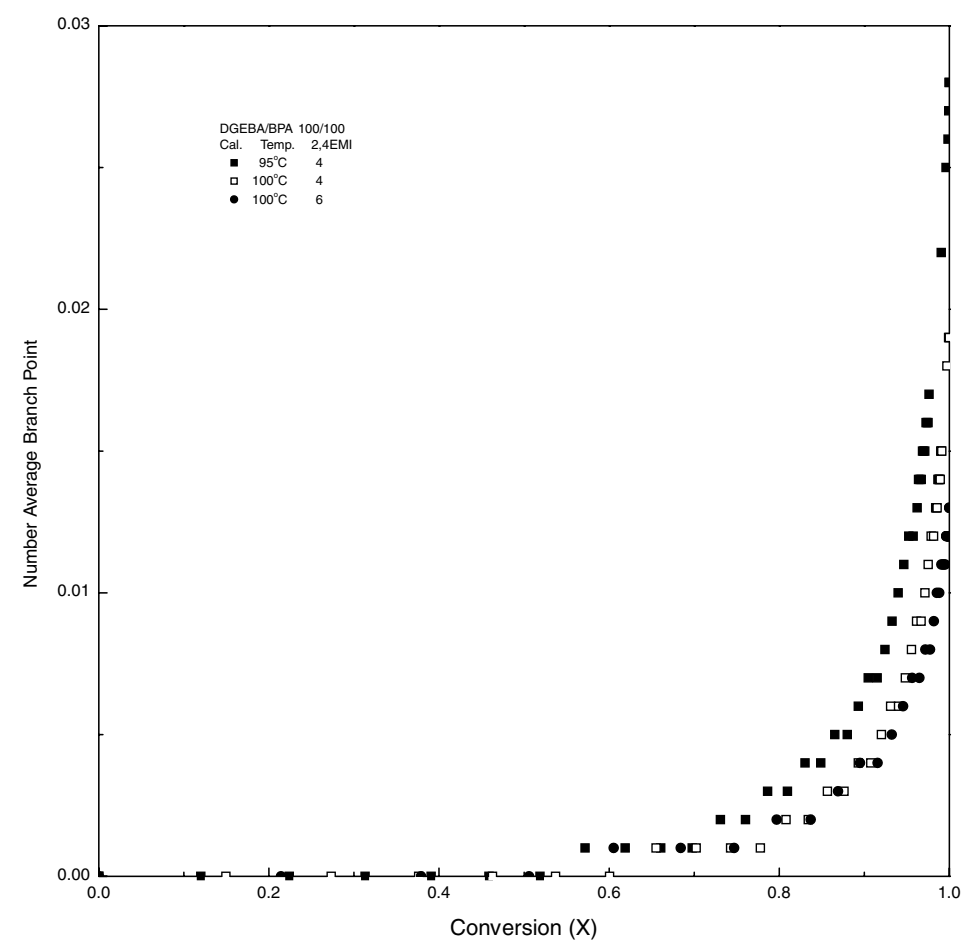

Fig. 6. The number average branch point of the sol part vs. conversion for DGEBA cured with $100 \%$ molar ratio of BPA at different reaction temperatures and 2,4EMI molar ratios. 


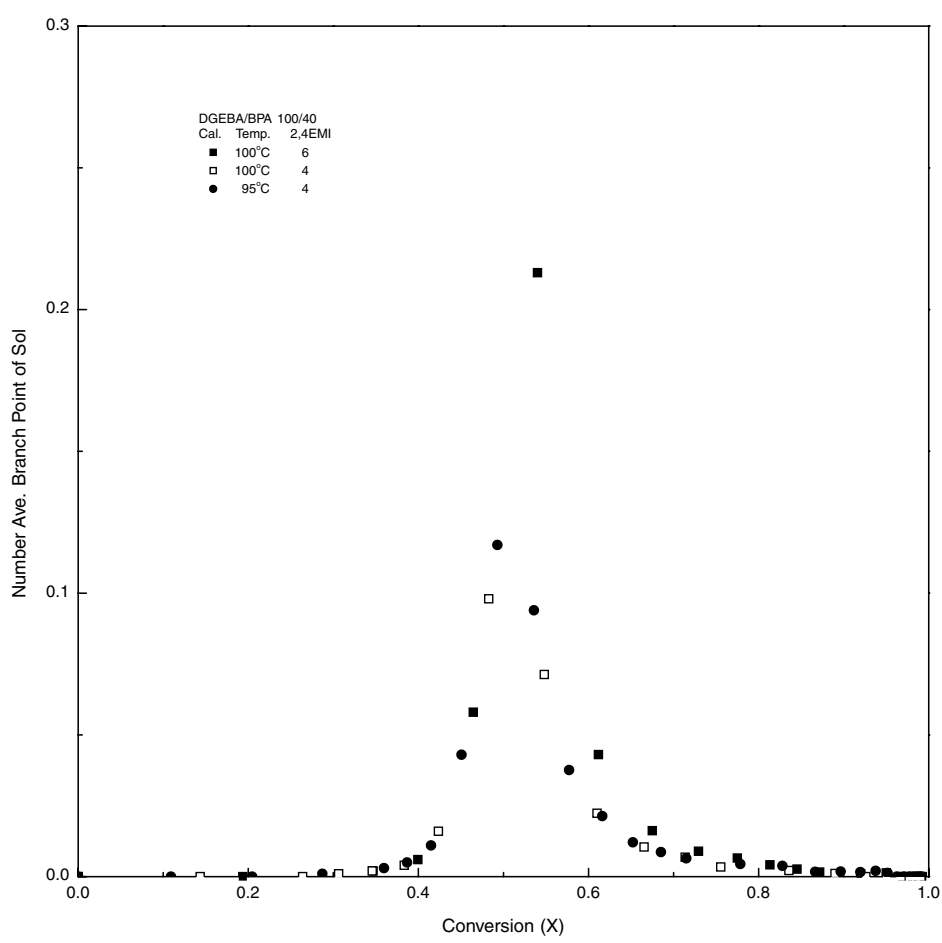

Fig. 7. The number average branch point of the sol part vs. conversion for DGEBA cured with $40 \%$ molar ratio of BPA at different reaction temperatures and 2,4EMI molar ratios.

weight $\left(\bar{M}_{\text {red }}\right)$, which represented the molecular weight of the linear phantom molecules that possessed the same hydrodynamic volume as the branched ones.

Fig. 8 shows the molecular weight profile vs. conversion, when the BPA molar ratio is $100 \%$. As most of the polymer chains are of linear structure at this condition, the absolute molecular weight $\left(\bar{M}_{\mathrm{w}}\right)$ is almost the same as the reduced molecular weight $\left(\bar{M}_{\text {red }}\right)$. The profiles of the calculated

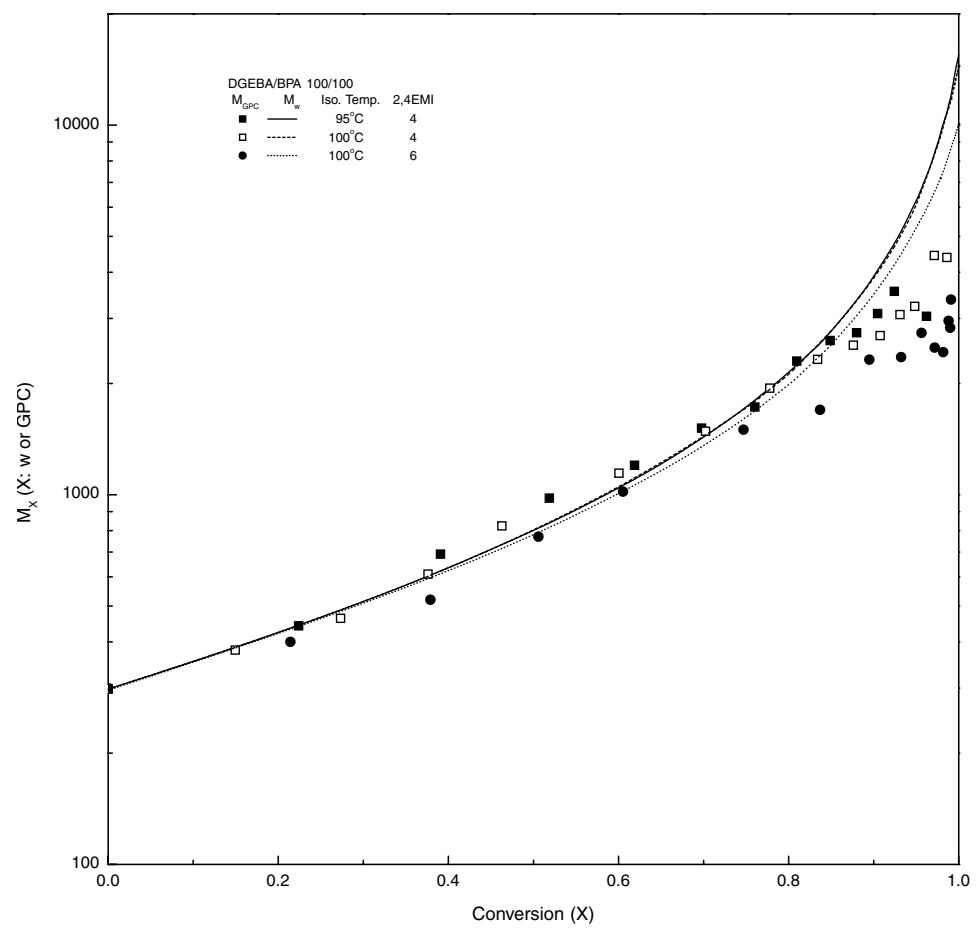

Fig. 8. The comparison of $\bar{M}_{\mathrm{GPC}}, \bar{M}_{\mathrm{w}}$, and $\bar{M}_{\text {red }}$ vs. conversion for DGEBA cured with $100 \%$ molar ratio of BPA at different 2,4EMI molar ratios and reaction temperatures. 


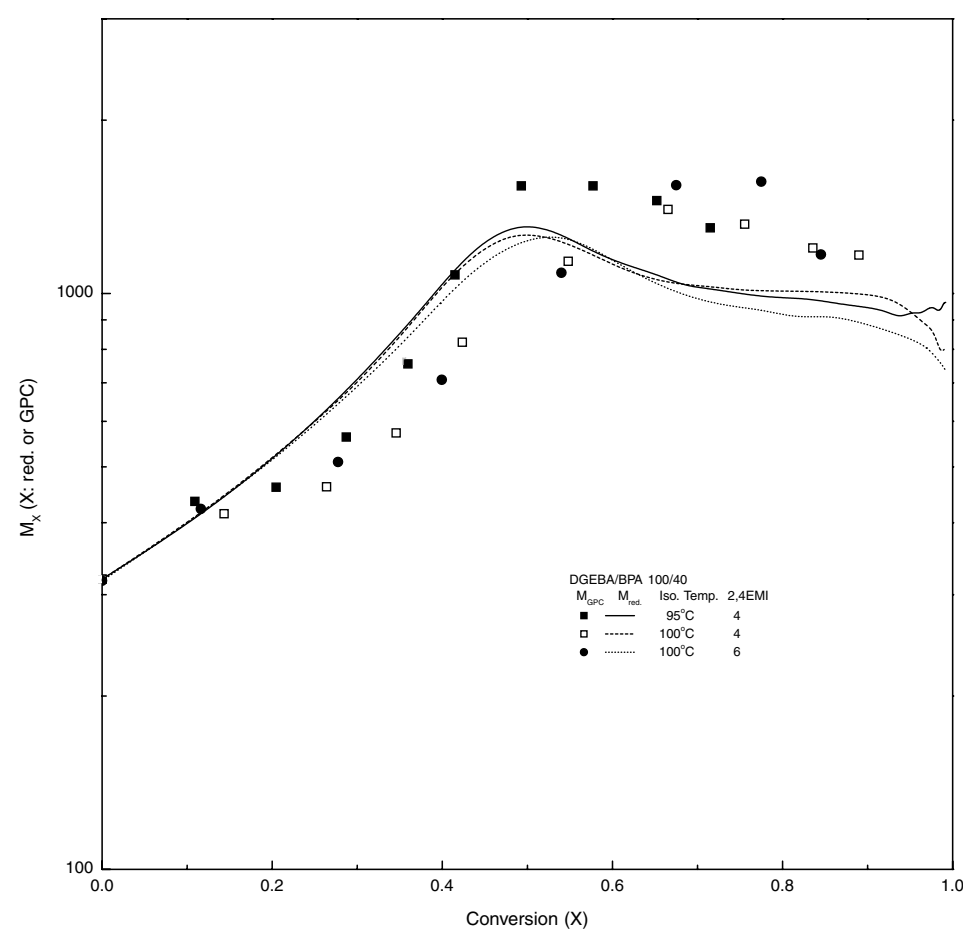

Fig. 9. The comparison of $\bar{M}_{\mathrm{GPC}}$ and $\bar{M}_{\text {red }}$ vs. conversion for DGEBA cured with $40 \%$ molar ratio of BPA at different 2,4EMI molar ratios and reaction temperatures.

results coincide with the GPC experimental data $\left(\bar{M}_{\mathrm{GPC}}\right)$ very well before the diffusion effect becomes serious. It is found that both the reaction temperature and molar ratio of 2,4EMI have no obvious effect on the profiles.

A comparison of reduced molecular weights of the sol part and the experimental data by GPC as the BPA molar ratio is lowered to $40 \%$, is plotted in Fig. 9. The reaction

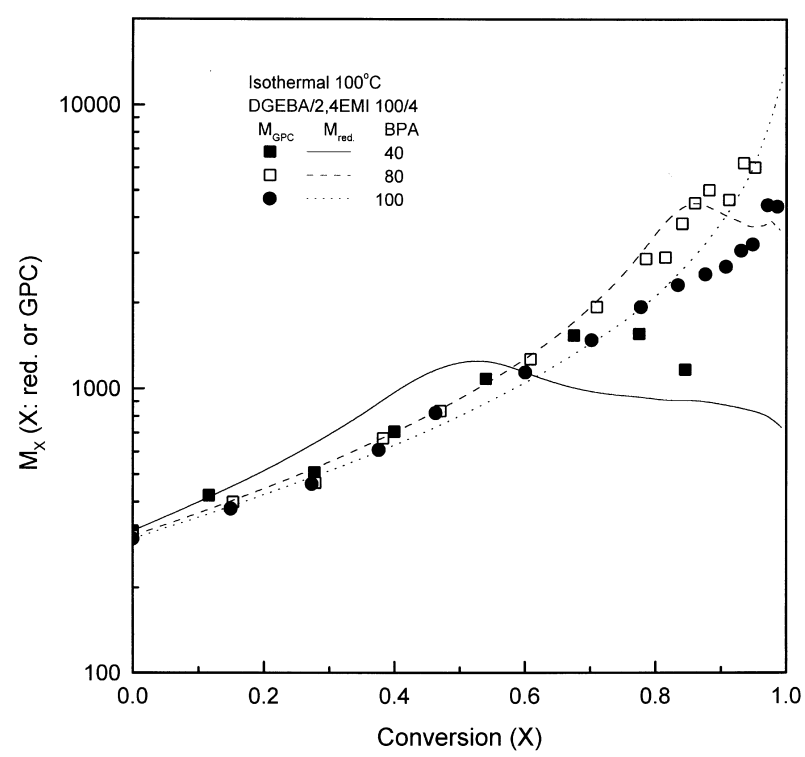

Fig. 10. The comparison of $\bar{M}_{\mathrm{GPC}}$ and $\bar{M}_{\text {red }}$ vs. conversion for DGEBA cured with $4 \%$ molar ratio of 2,4EMI and different BPA molar ratios at $100^{\circ} \mathrm{C}$. temperature and the molar ratio of 2,4EMI have only a little influence on the molecular weight profiles. Both the reduced molecular weights by the MC simulation and those by the GPC analysis coincide with each other quite well.

The influence of BPA molar ratio on the molecular weight profile of the sol part is shown in Fig. 10. The BPA molar ratio is the most effective factor dominating the molecular weight profiles since the BPA molar ratio controls the beginning of gel formation, the structural type of polymer chains, i.e. linear or branched, and the branch or loop density in the gel part. Both the MC simulation and the experimental data coincide with each other very well before the diffusion effect becomes serious.

The glass transition temperatures $\left(T_{\mathrm{g}}\right)$ of the final cured resins are listed in Table 4 . The reaction temperature does not change the $T_{\mathrm{g}}$ of the cured resins from the DSC analysis, and the 2,4EMI molar ratio has only a slight influence on the $T_{\mathrm{g}}$ of the cured resins. The results of $T_{\mathrm{g}}$ by the DSC agree with the findings in structural properties; i.e. the BPA molar ratio is the most important factor to control the final properties of the cured epoxy resin. Neither the reacting temperature nor the 2,4EMI concentration has much influence on the structural properties.

\subsection{Branch point and loop density of the gel part}

According to Flory's studies [13], the branch and loop density used here are defined as the total branch points and loop numbers in the gel part divided by the weight of the gel part. The density profiles of the gel part are shown in Fig. 11. 


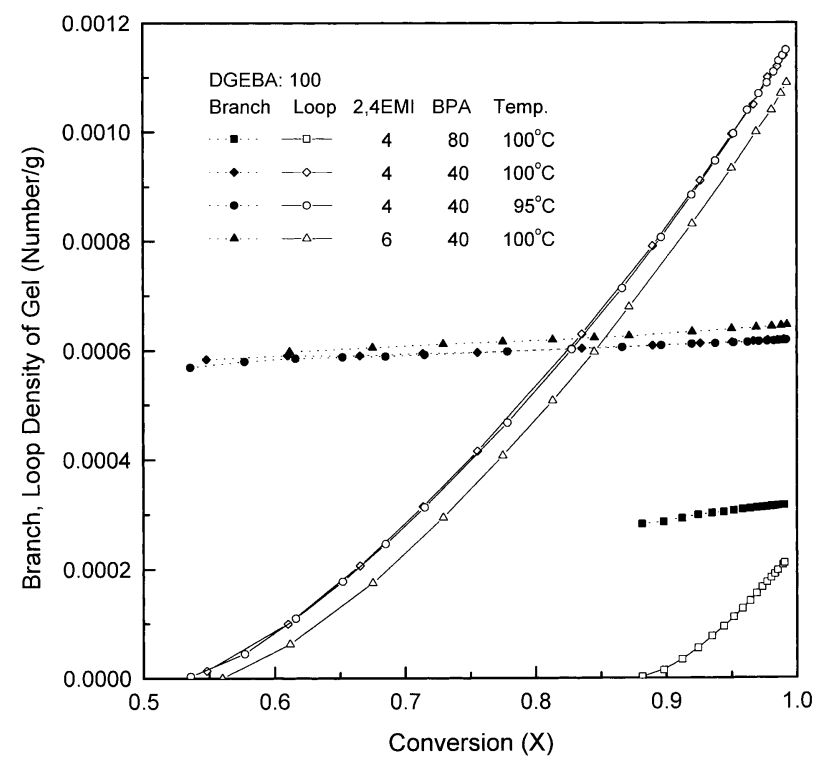

Fig. 11. The branch density and loop density of the gel part vs. conversion for DGEBA cured at different reaction conditions.

It is found that the branch densities increase slightly with the increasing conversion; however, the loop densities grow up sharply after the gel point. As the gel parts are formed, the molecules remaining in the sol part connect onto the gel molecule through the inter-chain $\mathrm{R}-\mathrm{O}^{-}$-epoxide reaction and new branch points build up simultaneously. Fig. 5, shows that the weight fraction of the gel part increases sharply after the critical conversion. It means that few chances of the inter-chain R$\mathrm{O}^{-}$-epoxide reaction occur between the sol and gel part; therefore, the branched density in the gel part increases slightly. Meanwhile, a lot of unreacted epoxide groups on the gel part go through the intra-chain $\mathrm{R}_{-} \mathrm{O}^{-}$-epoxide reaction, which increases the number of loops in the gel part. Thus the loop density increases sharply after the critical conversion as shown in Fig. 11. The reaction temperature does not affect the profiles of branch or loop density significantly. As a higher molar ratio of 2,4EMI is used, the gel fraction becomes slightly smaller at the same epoxide conversion as shown in Fig. 5. This means that more molecules exists in the sol part after the gel point, which increases the chance of inter-chain $\mathrm{R}^{-} \mathrm{O}^{-}$-epoxide reaction but decreases the intra-chain $\mathrm{R}-\mathrm{O}^{-}$-epoxide reaction inside the gel. Hence, increasing the molar ratio of 2,4EMI slightly lowers the loop density but raises the branch density of the gel part. As the BPA molar ratio is increased, more phenol molecules participate in the epoxide-phenol reaction, which build up more linear chains and the gel point is delayed. In other words, less epoxide groups remain and take part in the following inter-chain or intra-chain R$\mathrm{O}^{-}$-epoxide reactions. Inasmuch, the values of branch density and loop become smaller as the BPA molar ratio increases from 40 to $80 \%$.

\section{Conclusions}

In this work, the structural properties of imidazole cured epoxy-phenol resins were simulated by the MC method. The influences of BPA, 2,4EMI molar ratio, and the reaction temperature were discussed. Moreover, the theoretical results were verified experimentally by the DSC, sol extraction test, and GPC results.

The BPA molar ratio was the most important factor to dominate the structural properties of the cured resins. When the BPA molar ratio was $100 \%$ relative to DGEBA, there were enough phenol groups to react with epoxide groups. Thus, most of the cured molecules were of a linear structure, and there was no gel part formed throughout the whole reaction. When the BPA molar ratio was lower than $100 \%$, the branched structure formed through the $\mathrm{R}_{-} \mathrm{O}^{-}$ epoxide reaction as the phenol groups were nearly consumed. The gel part was built up after a critical conversion, and lesser the molar ratio of BPA was used, the earlier the critical conversion was reached. But the 2,4EMI molar ratio and the reaction temperature had no obvious influence on the structural properties of the cured resins. As the structural properties of the gel part were considered, the branch density and loop density were mainly determined by the molar ratio of BPA. The more the BPA was used, the lesser epoxide groups participated in the inter-chain and intrachain $\mathrm{R}^{-} \mathrm{O}^{-}$-epoxide reaction. Thus, the branch and loop density decreased with rising BPA molar ratio. However, the reaction temperature and the 2,4EMI molar ratio had little influences on the formed gel part.

\section{References}

[1] Kinjo N, Ogata M, Nishi K, Kaneda A. Adv Polym Sci 1989;88:1-59.

[2] Soane DS, Martynenko Z. Polymers in microelectronics. New York: Elsevier, 1989.

[3] Wong CP. Polymers for electronic and photonic applications. New York: Academic Press, 1993.

[4] Shechter L, Wynstra J. Ind Eng Chem 1956;48(1):86-93.

[5] Gagnebien D, Madec PJ, Marechal E. Eur Polym J 1985;21(3):27387.

[6] Biernath RW, Soane DS. Contemporary topics in polymer science, 7. New York: Plenum Press, 1992. p. 103-59.

[7] Chen YC, Chiu WY, Lin KF. J Polym Sci Polym Chem Ed 1999;37:3233-42.

[8] Chen YC, Chiu WY. Macromolecules 2000;33:6672-84.

[9] Chen, YC. PhD dissertation, National Taiwan University, Taiwan, 2000.

[10] Shy LY, Leung YK, Eichinger BE. Macromolecules 1985;18:983-6.

[11] Turi EA. Thermal characterization of polymeric materials. New York: Academic Press, 1981 (chap. 5).

[12] Barton JM. Adv Polym Sci 1985;72:112-51.

[13] Flory PJ. Principles of polymer chemistry. Ithaca, NY: Cornell University Press, 1953. 